\title{
UN “CORAZÓN” EN EL DESIERTO. INDAGACIONES EN TORNO A LA PROPUESTA URBANA DE LA VILLA EL CHOCÓN EN LA PATAGONIA ARGENTINA (1968/1972)
}

DOI: https://doi.org/10.18861/ania.2019.9.2899

Dra. Arq. Cecilia Parera

ORCID iD: http://orcid.org/0000-0002-9880-4896 


\section{DRA. ARQ. CECILIA PARERA}

Doctora en Arquitectura, Universidad Nacional de La Plata (Argetina). Master in Architecture, University of Utah (Estados Unidos). Arquitecta, Universidad Nacional del Litoral (Argentina). Profesora de grado y posgrado, e investigadora de la Facultad de Arquitectura, Diseño y Urbanismo en el área de ciencias sociales, Universidad Nacional del Litoral (Argentina).

FECHA DE RECEPCIÓN: 1 de abril de 2019.

FECHA DE ACEPTACIÓN: 8 de mayo de 2019.

REGISTRO BIBLIOGRÁFICO: PARERA, C. (2019). Un “corazón" en el desierto. Indagaciones en torno a la propuesta urbana de la Villa el Chocón en la Patagonia argentina (1968/1972). Anales de Investigación en Arquitectura, 9 (1), 25-44. 


\section{RESUMEN}

En los años cincuenta y sesenta del siglo XX se difundió una crítica disciplinar sobre la ciudad existente, rechazando los postulados funcionalistas aplicados en la entre-guerra, en beneficio de lecturas atentas a la percepción fenomenológica del espacio, a la mixtura de usos y a la escala humana. El artículo pretende indagar en torno a este cambio de paradigma disciplinar, adoptando a la Villa el Chocón en la Patagonia argentina, y particularmente a la propuesta para su centro cívico, como principal caso de estudio, siendo que este pequeño poblado pone en evidencia un nuevo abordaje proyectual. En particular, la hipótesis del trabajo plantea una proximidad conceptual de los autores de la mencionada obra con los postulados que fundamentaron el alejamiento del llamado Movimiento Moderno, así como una adhesión al lema "el corazón de la ciudad", cuestiones debatidas en sede europea desde principios de la década de 1950. En atención a los objetivos planteados, el análisis históricocrítico del caso seleccionado ha permitido identificar estas afinidades, diferenciadas tanto del racionalismo como estrategia predominante hasta el estallido de la Segunda Guerra Mundial, como el posterior abordaje sistémico e indeterminado que caracterizaría a gran parte de la producción arquitectónica de los años 1970 en Argentina.

Palabras Clave: Arquitectura, planificación urbana, Argentina.

\section{ABSTRACT}

In the fifties and sixties of the twentieth century, a disciplinary critique of the existing city was disseminated, rejecting the functionalist postulates applied in the inter-war, for the benefit of careful readings of the phenomenological perception of space, the mixture of uses and the human scale The present article pretends to investigate around this change of disciplinary paradigm, adopting the Villa el Chocón in the Argentinian Patagonia, and particularly the proposal for its civic center, as the main case of study, being that this small town puts in evidence a new approach regarding project strategies in urban and architectural matters. In particular, the hypothesis that guides the paper raises a conceptual proximity of the authors of the aforementioned work with the postulates that supported the distancing of the so-called Modern Movement, as well as an adherence to the motto "the heart of the city", issues debated in Europe since the early 1950s. In attention to the stated objectives, the historical-critical analysis of the selected case made it possible to identify these affinities, differentiated as much from rationalism as the predominant strategy until the outbreak of the Second World War, as well as the subsequent systemic and indeterminate approach that would characterize a large part of the architectural production of the 1970s in Argentina.

Keywords: Architecture, urban planning, Argentina. 


\section{PRIMEROS INDICIOS DEL DESENCANTO CON EL PARADIGMA MODERNO}

Las negativas consecuencias sociales, políticas y económicas de la Segunda Guerra Mundial causaron un cisma de significativa envergadura en el pensamiento moderno, el que comenzó a ser sistemáticamente cuestionado en la inmediata posguerra. Diversas corrientes de pensamiento se centraron en la condición del hombre, como el existencialismo, el humanismo y la fenomenología, de la mano de intelectuales como Martin Heidegger, Theodor Adorno y Gasset o Jean-Paul Sartre (Montaner, 1997). Estos posicionamientos marcaron fuertemente al campo de la arquitectura y el urbanismo, constituyéndose en fundamentos epistemológicos para la crítica al estricto funcionalismo del período de entre-guerras. Ante la negación sistemática de la tradición y del carácter por parte de la producción arquitectónica "moderna”, Sigfried Giedion manifestaba "la necesidad de una Nueva Monumentalidad" $(1944)^{1}$, debiendo para ello crear continuidad entre el pasado, el presente y el futuro, así como brindar a la experiencia humana colectiva una expresión simbólica al inscribirla en los tejidos urbanos existentes. Bajo esta mirada la estructuración de la ciudad

1 Esta demanda por una "nueva monumentalidad" buscaba contraponerse a la "muerte del monumento" declarada por Lewis Mumford en 1937 al reconocer que la arquitectura moderna, por su propia naturaleza dinámica, no podía ser plasmar una época con las mismas estrategias representativas que se habían concretado los edificios de la antigüedad. en cuatro funciones básicas, habitar, trabajar, circular y recrearse, demostraba ser insuficiente (Mumford, 2000). Este ordenamiento, delineado en el Congreso Internacional de Arquitectura Moderna (CIAM) IV desarrollado en Atenas en 1933, guió gran parte de las propuestas urbanísticas de estos años, como el Plan Maciá para Barcelona (GATEPAC, Le Corbusier y Pierre Jeanneret, 1932/1935) y el Plan Director para Buenos Aires (Le Corbusier, Jorge Ferrari Hardoy y Juan Kurchan, 1937/1938).

En esta línea, el arquitecto alemán Paul Zucker también reparaba en la generalizada desatención por cuestiones vinculadas a la representación cívica.

A pesar que la lógica funcional se ha convertido en nuestra ley arquitectóncia suprema, debemos aún superar sus deficiencias mediante el impacto emocional de la individualidad creativa... a través de un profundo interés y una comprensión completa de los problemas sociales básicos

(Zucker, 1944:9).

Los mencionados virajes epistemológicos incidieron en particular en los debates del CIAM, cuyos miembros volvieron a reunirse en Bridgewater en 1947, tras diez años de interrupción por el conflicto bélico. Como propuesta general un número importante de sus miembros indicaron la necesidad de "trabajar por la creación de un ambiente físico que satisfaga las necesidades emocionales y materiales del 


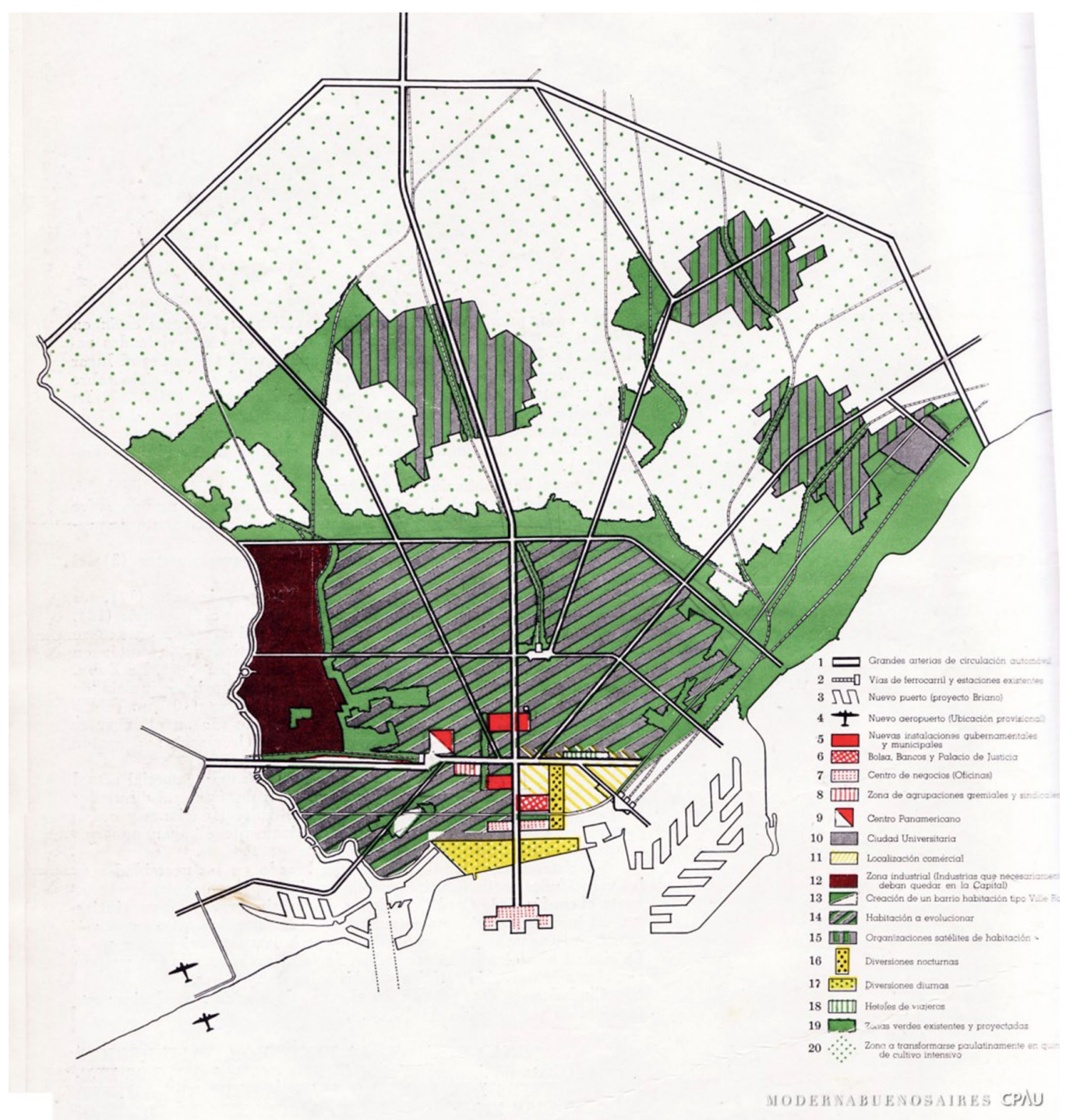

Figura 1.

Plan Director para Buenos Aires (Le Corbusier, Jorge Ferrari Hardoy y Juan Kurchan, 1937/1938). 


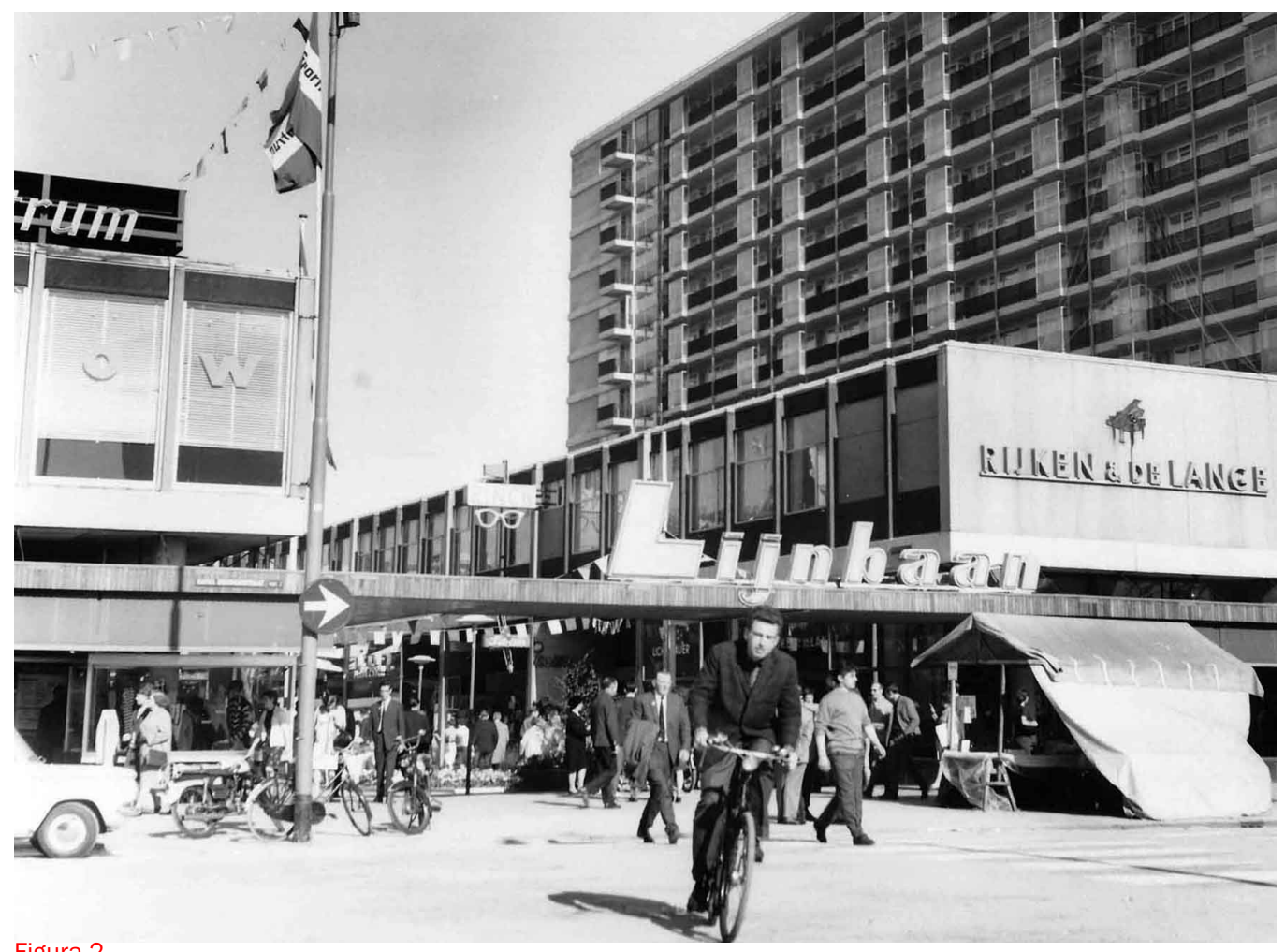

Figura 2.

Vista peatonal del Distrito Lijnbaan en Rotterdam, 1965 (Jaap Bakema y Jo van der Broek, 1949/1953).

hombre, y estimular su crecimiento espiritual" (Giedion, 1951: 17), lo que en el contexto de la segunda posguerra implicaba abordar una serie de cuestiones contingentes, como los procesos de metropolización, la tugurización de sectores funcionalmente especializados y la recuperación de áreas devastadas durante la Segunda Guerra Mundial.

La reconstrucción de las ciudades afectadas por los bombardeos constituyó uno de los temas centrales del evento, planteándose la necesidad de definir lineamientos respecto de dónde ubicar los nuevos equipamientos, cuáles áreas históricas debían ser preservadas, cómo debían organizarse funcionalmente los sectores urbanos, y qué densidad de ocupación era conveniente, entre otros aspectos. El distrito comercial Lijnbaan en Rotterdam, proyectado por los arquitectos holandeses Jaap Bakema y Jo van der Broek en 1949 para un área central de la ciudad destruida por los ataques alemanes, constituye uno de los ejemplos más tempranos del nuevo posicionamiento. 
El conjunto se inserta sutilmente en la trama urbana existente, recuperando el valor de la calle corredor como eje de las relaciones sociales. A su vez, plantea un programa de usos mixtos -comercios, viviendas, oficinas y estacionamiento- relacionados a partir de espacios públicos peatonales. La altura de las edificaciones se adecúa a las distintas relaciones de escala presentes, incorporando mobiliario urbano -como marquesinas, pérgolas, maceteros y bancos- para promover transiciones, articulaciones y áreas de reparo (Van den Heuvel, 2017).

El definitivo alejamiento de los postulados funcionalistas se verificó en el CIAM VIII, concretado en Hoddston en 1951. Las áreas centrales fueron redefinidas bajo el lema de “El corazón de la ciudad". La referencia a este órgano vital se caracterizaba por una doble naturaleza; por un lado, como símbolo, ya que refería a los sentimientos -sin validación racional-, y, por otro, como metáfora de centro de donde emanaban las energías (Zuccaro Marchi, 2013). En este evento el arquitecto español Josep Lluís Sert, presidente de los CIAM por estos años, afirmaba que era "necesaria la planificación de los sectores centrales de las ciudades y el desarrollo de nuevos centros donde la vida urbana cree lugares de reunión" (Sert, 1955a: VI), "estos núcleos actuarán de elementos catalizadores, y alrededor de los mismos se desenvolverá la vida de la comunidad; en ellos se agruparán los edificios públicos de distinta clase" (Sert, 1955b: 6).
No es de desdeñar que por fuera del CIAM estas temáticas también agitaban otras preocupaciones, como aquella en torno a la percepción fenomenológica de la arquitectura por parte del arquitecto noruego Christian Norberg-Schulz, en relación a los estragos generados por los proyectos de renovación urbana de la activista canadiense Jane Jacobs, y en atención a la incapacidad lingüística e identitaria de las propuestas racionalistas de la mano del arquitecto estadounidense Robert Venturi y la arquitecta zambiana Denisse Scott Brown.

La disponibilidad de revistas extranjeras, la pronta traducción al castellano de los textos que abordaban estas temáticas, la prolífica red de relaciones personales e intercambios epistolares entre profesionales, y los asiduos viajes de especialistas permiten inferir un nivel de vinculación considerable de los profesionales latinoamericanos con los debates vigentes. Los espacios colectivos significativos propuestos por el arquitecto franco-colombiano Rogelio Salmona, la atención por los aspectos materiales y sensibles de los proyectos de la arquitecta italo-brasilera Lina Bo Bardi, y la recuperación de la voluntad simbólica a partir de volumetrías contundentes y la iluminación escenográfica de los espacios del arquitecto argentino Claudio Caveri constituyen algunos de los tantos exponentes de la "reacción antirracionalista” en Latinoamérica (Iglesia, 1978). 


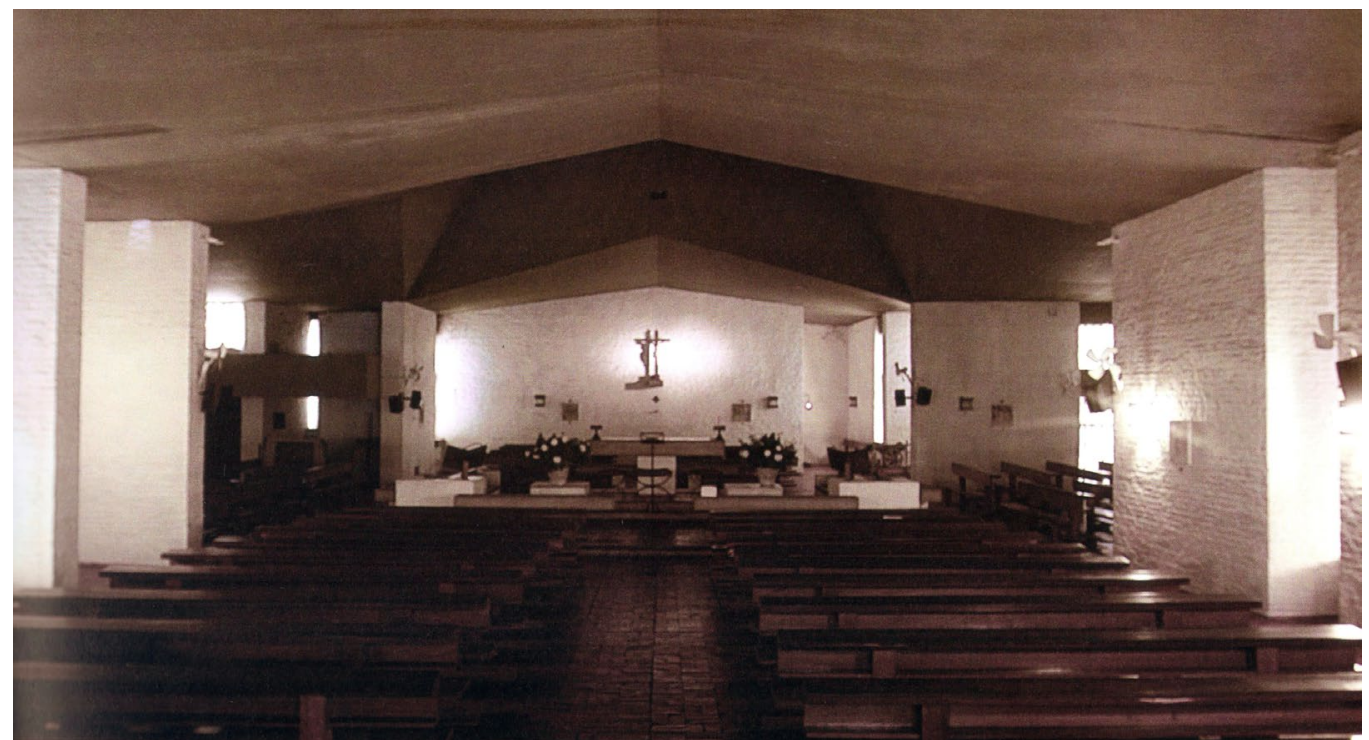

Figura 3.

Interior de la Iglesia Nuestra Señora de Fátima en Martínez, Provincia de Buenos Aires, 2005 (Claudio Caveri y Eduardo Ellis, 1956/1959).

\section{UN NUEVO PARADIGMA: LA CIUDAD COMO COBIJO DE LA VIDA DE LA COMUNIDAD}

La Villa permanente El Chocón en la Provincia argentina de Río Negro, planificada para alojar al personal del complejo Hidroeléctrico El Chocón - Cerros Colorados², constituye un caso paradigmático en el marco del mencionado debate disciplinar. Esta pequeña

2 Esta infraestructura formó parte del amplio plan de mejora del servicio energético impulsado por el Estado desarrollista en Argentina en la década de 1960. Tras la creación de la empresa de mayoría estatal Hidroeléctrica Norpatagónica S.A. en 1967 se desarrolló el proyecto completo con celeridad, contando con asesores y financiamiento internacionales. Sobre el tema, ver Bandieri y Blanco 2012. ciudad ex novo fue proyectada en 1968 por dos estudios de arquitectos de Buenos Aires, Llauró y Urgell, y Antonini, Schön, Zemborain y Firpo, constituyéndose en una oportunidad única en términos proyectuales para marcar su posicionamiento. "Para nosotros la crítica al mundo contemporáneo y sus productos no terminaba en un rechazo absoluto, sino más bien en la necesidad de comenzar una lucha por modificarlo" (Llauró y Urgell, 1973, p. 29).

Un antecedente cercano geográficamente en el marco de este programa urbanístico podrían haber sido los poblados extractivos asociados al petróleo, como Callacó en Argentina, o bien a la minería, como Puerto Cristal en Chile, pero ya habían sido fuertemente criticados por la 


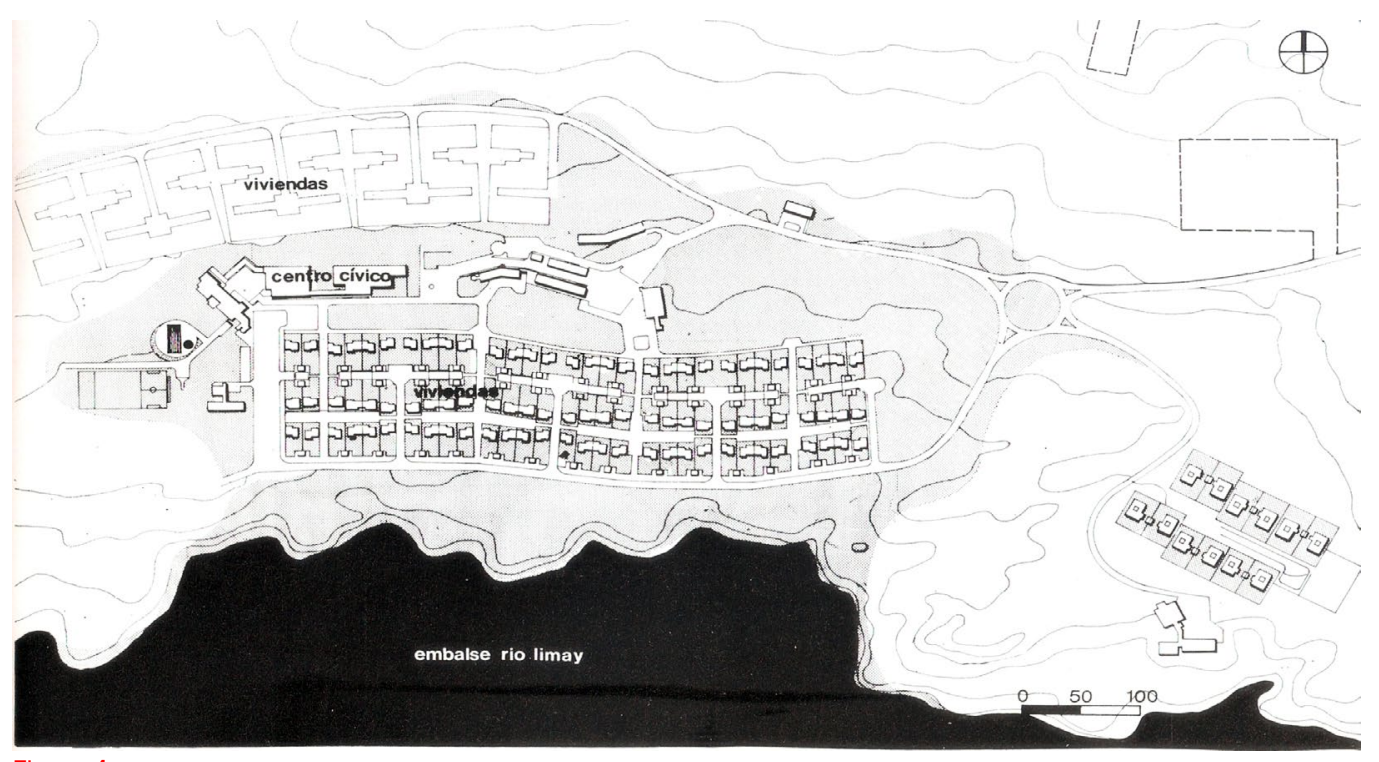

Figura 4.

Interior de la Iglesia Nuestra Señora de Fátima en Martínez, Provincia de Buenos Aires, 2005 (Claudio Caveri y Eduardo Ellis, 1956/1959).

deficiente calidad constructiva de las viviendas y los espacios comunes, la segregación según escala laboral de sus habitantes, así como la ausencia de áreas públicas, cuestiones que promovían una alta conflictividad social (Williams, 2014b) ${ }^{3}$. Por estos años las new towns británicas, propuestas en 1946 para mitigar la sobrepoblación que sufría la ciudad de Londres, constituían un referente más afín en términos conceptuales, ya que planteaban unidades poblacionales autónomas, de baja densidad y con equipamiento suficiente para satisfacer sus necesidades comunales

3 Estos modelos urbanos, que se iniciaban como meros campamentos, replicaban las estrategias impulsadas por los emprendedores industriales durante la primera mitad del siglo XIX, quienes contruían company towns, como Withnell Fold en Inglaterra y Lowell en Estados Unidos.

básicas. La organización de los poblados de Hook (1958) y Runcorn (1964) -partes del mencionado plan de desconcentración inglésreforzaba el rol cívico del área central y definía nuevas áreas residenciales de baja densidad a partir de un patrón lineal y centrífugo. Por estos años, varias revistas especializadas argentinas, como Summa y Nuestra Arquitectura, dieron amplia difusión a estas propuestas urbanísticas y a las ideas que las sustentaban, y bibliotecas como la de la Sociedad Central de Arquitectos y de la Facultad de Arquitectura y Urbanismo de la Universidad de Buenos Aires contaban con numeroso material sobre estos temas.

La localización de la Villa el Chocón permite reconocer debates contemporáneos sobre la necesidad de considerar los valores 
psicológicos de la percepción del entorno, en sintonía con el carácter concreto, empírico y existencial asociado al concepto de lugar (Norberg Schulz, 1979). Esta ubicación fue determinada en atención a las particularidades del entorno geográfico, eligiéndose una meseta natural frente al futuro embalse, protegida de los persistentes vientos de la región. Por su parte, el esquema general de la villa organizaba las actividades en sectores lineales identificables, los que podían crecen en sus extremos sin afectar al resto, tomando como pauta una baja densidad de ocupación. A su vez, se buscó independizar la circulación peatonal de la vehicular, promoviendo a la primera como principal conector de los accesos principales a los edificios públicos y a las viviendas, mientras que la segunda vinculaba los accesos de servicio. La configuración de esta doble circulación alejó el agrupamiento de la morfología del damero tradicional, asociándose más bien a las propuestas suburbanas de baja densidad que se multiplicaban en las periferias norteamericanas así como a las ya mencionadas new towns inglesas. En esta línea, la inclusión de áreas recreativas con juegos para niños en los cruces de circulaciones peatonales contribuyó a diversificar la experiencia de los recorridos.

El programa de necesidades de la villa exigía proveer alojamiento a cerca de doscientas familias - uno 500 habitantes- en viviendas de dos y tres dormitorios, cuya construcción se realizaría en dos etapas. La tipología de unidad adoptada se desarrollaba de manera individual y en una única planta, con una superficie generosa, organizándose a partir de un ingreso central que separaba el área de dormitorios del área pública, y, más allá de ésta, el área de servicios. Evidentemente, la holgura con la que fue posible resolver las células distaba a esta propuesta de los debates vigentes en Argentina sobre las políticas de vivienda por parte del Estado Nacional, tendientes a la agrupación multifamiliar, a la compactación de la planta y a la alta densidad. A su vez, uno de los principales desafíos planteados a los arquitectos a cargo fue dar privacidad a los pobladores, ya forzados a relacionarse permanentemente en su trabajo, por lo que las viviendas fueron proyectadas asegurando cierto nivel de aislación a partir de la dimensión generosa de sus lotes, la parquización de las medianeras, y la ubicación y orientación de las aberturas (Introducción, 1970).

\section{EL CENTRO CÍVICO COMO “CORAZÓN" DE LA VIDA EN COMUNIDAD}

En paralelo a las tiras residenciales, y algo retirado, fue ubicado el centro cívico de la villa, donde se alojarían los edificios institucionales y las actividades públicas (Plotkin, 2018).La propuesta de este centro cívico claramente remitía al espíritu planteado en el marco del debate en torno al "corazón de la ciudad", como identificaban las memorias de la obra. "El objetivo principal fue el recrear un ámbito con la riqueza necesaria para el desarrollo pleno de la vida individual y colectiva" (Llauró y Urgell, 1978: 46). 


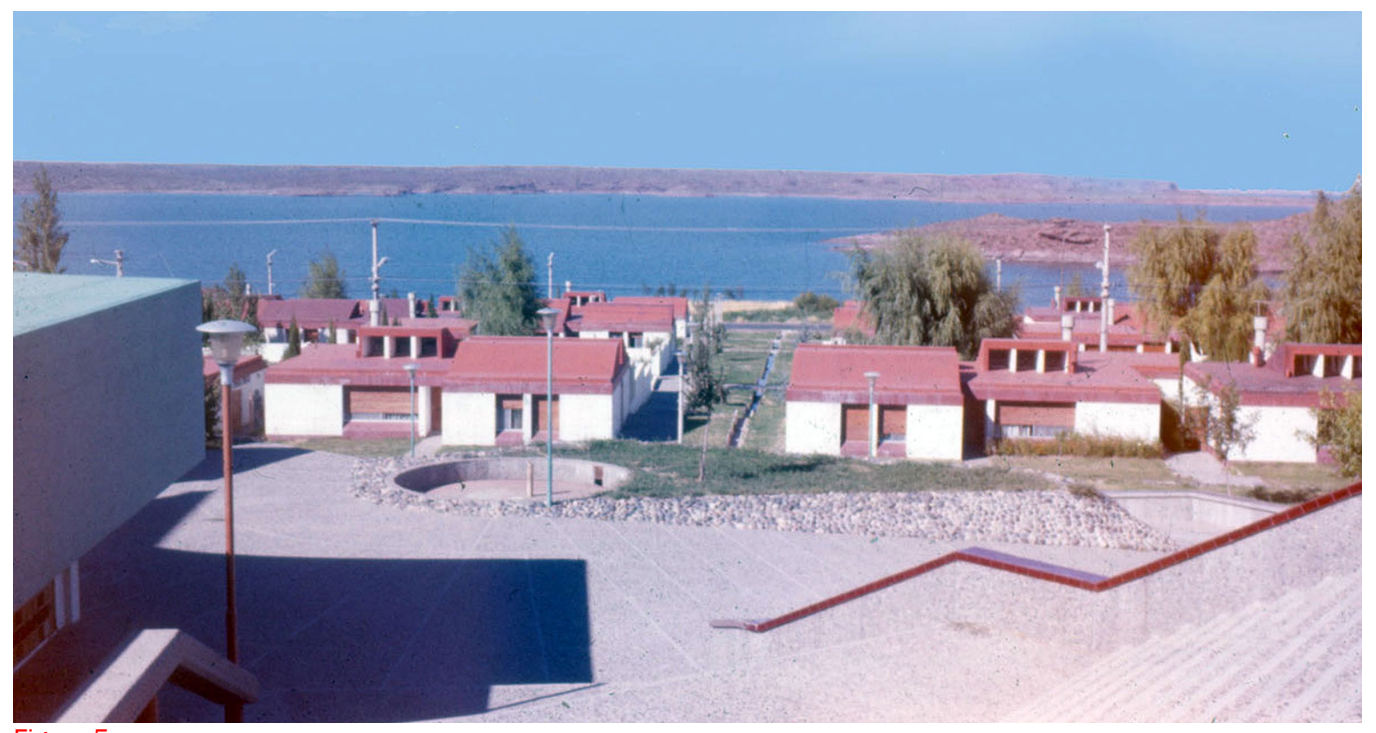

Figura 5.

Sector residencial de la Villa el Chocón, vista desde el extremo Este del centro cívico, ca. 2008.

Emplazado en un banco natural con vistas al lago por sobre las viviendas, los edificios públicos requeridos por el comitente fueron organizados linealmente a lo largo de una calle peatonal. "Se buscó, mediante dimensiones ajustadas, obtener un lugar que acentuara la concentración y el movimiento de gente" (Villa, 1970: 52). Frente a los distintos edificios representativos la calle peatonal se ensancha para promover espacios de congregación, y es amenizada con bancos, escalinatas, parquizado y juegos, mientras que frente a los comercios y el centro comunal el camino se estrecha y es protegida por pérgolas cubiertas por láminas traslúcidas. En particular, el club deportivo, el salón de usos múltiples y la escuela fueron ubicados en el extremo Oeste, asegurando su funcionamiento de manera integrada y multiplicando sus posibilidades de apropiación como cine, salón de fiestas, comedor, biblioteca, entre otras.

En el extremo Este del Centro Cívico la calle peatonal se amplía generando una plaza seca, desde la cual se accede, descendiendo una escalinata, al atrio de la iglesia. En línea con los planteos fenomenológicos en boga por estos años, los arquitectos a cargo reflexionaban que este proyecto “adquiere un grado alarmante de libertad. El diseño es entonces, casi exclusivamente, un lenguaje para afirmar una intención humana" (Llauró y Urgell, 1973: 28). Resulta de particular interés la resolución de la cubierta de este edificio religioso, una cáscara de hormigón armado invertida, la que se eleva hacia el lado más alejado para mantener la 


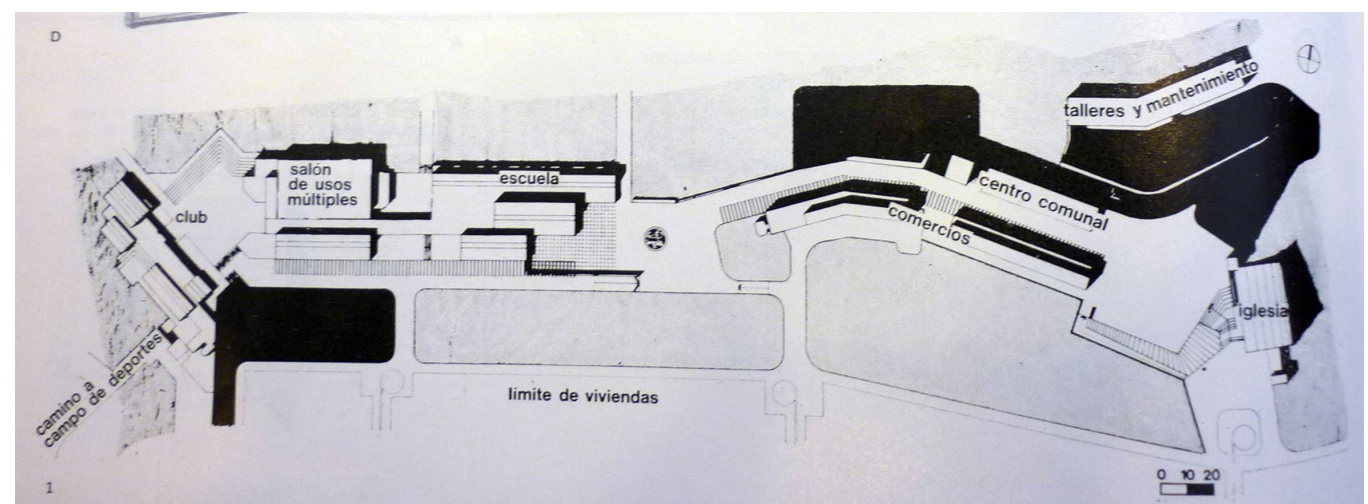

Figura 6.

Planta del centro cívico de la villa, 1968.

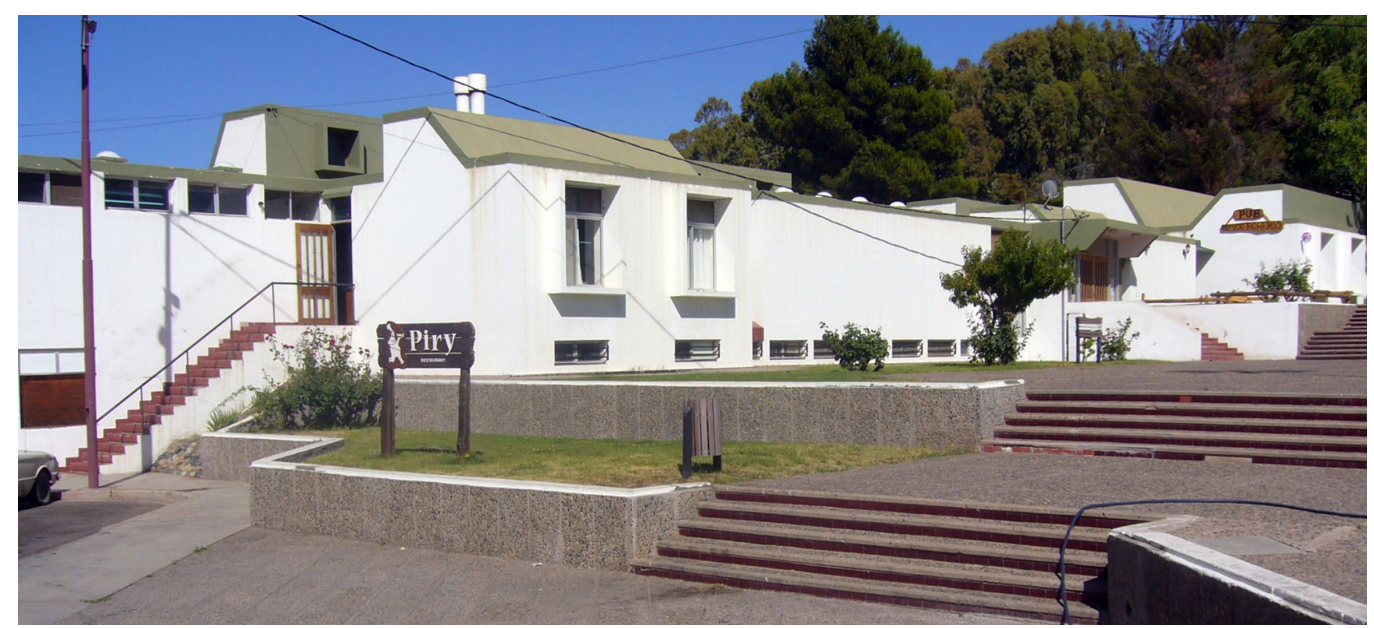

Figura 7.

Vista del centro cívico, circulación peatonal, 2007.

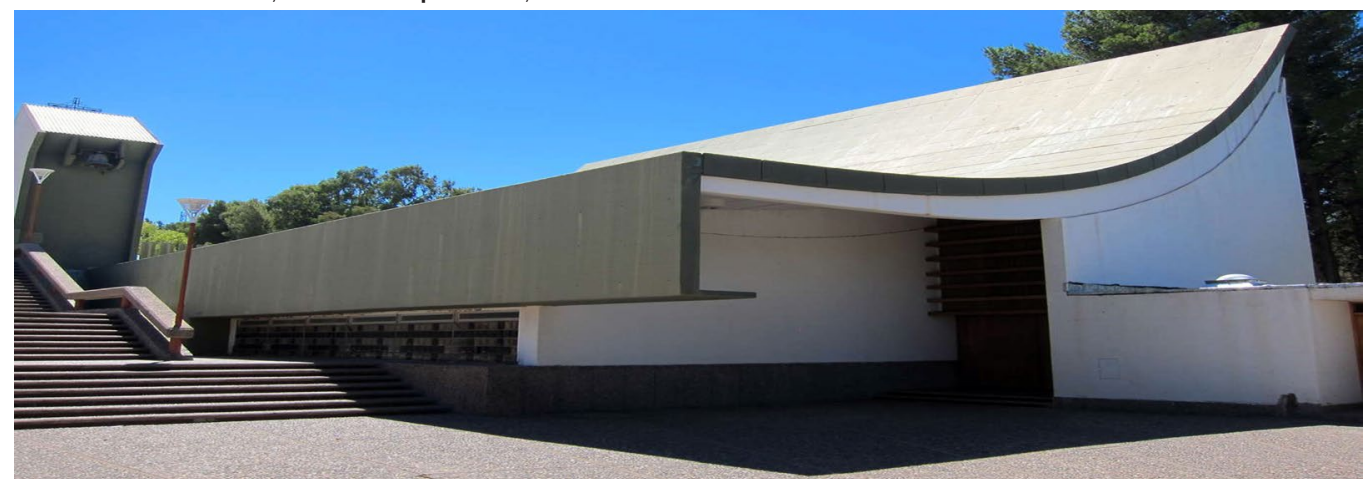

Figura 8.

Vista del atrio de la iglesia, 2010.

Anales de Investigación en Arquitectura Vol.9 | 2019 | № 1. Montevideo (Uruguay), 25-44 Universidad ORT Uruguay 
escala humana sin perder su naturaleza de edificio singular. La luz se transforma en un componente esencial en el ordenamiento espacial interior, ingresando difusamente a través de ventanales de ladrillos de vidrio coloreados y lucarnas de chapa translúcida. La planta, con tendencia centralizada, responde a una nueva línea de la liturgia católica en la que los fieles asumen un carácter protagónico, y que en el medio local ya había sido apelada en otras iglesias que por esos años alcanzaron gran difusión, como en la Parroquia Nuestra Señora de Fátima en Martínez, ilustrada en la imagen 03.

Este "corazón de la ciudad" no fue la única experiencia proyectual en torno a esta temática en Argentina. En esta línea de rechazo a la monotonía urbana impulsada por el funcionalismo es posible ubicar a las presentaciones ganadoras de los concursos para los centros cívicos de la Provincia de La Pampa (1955), del Partido de Tres de Febrero (1963) y de la ciudad de Berisso (1966). Estas propuestas ponen en evidencia las mencionadas preocupaciones, otorgando la centralidad del proyecto a la plaza de reuniones cívicas en el primer caso, estructurando subzonas para preservar la naturaleza específica de las actividades y de cada tipo de circulaciones en el segundo, y, en el último, graduando una sucesión de ámbitos públicos hacia el río a lo largo de una calle peatonal con la intención de fundirse con el entorno (Schere, 2008). La escala humana, las restricciones al automóvil y la inclusión de elementos espontáneos en el diseño urbano, entre otros aspectos, también formaron parte del sustento conceptual de estas intervenciones.

Retomando el caso patagónico, la premura por desarrollar la totalidad del proyecto en tres meses determinó la organización de equipos de trabajo que diseñaban cada edificio de manera individual, lo que en términos formales resultó una opción superadora, ya que logró transmitir una cierta heterogeneidad poco frecuente en estos proyectos a gran escala.

“El diseño de la villa recreó en un condensado espacio de tiempo y con el aporte de varios diseñadores trabajando en el marco de independencia, una cierta espontaneidad y un método aditivo en el proceso de proyecto, similar en alguna medida al desarrollo 'natural' de la conformación física de un pueblo comercial"

(Llauró y Urgell, 1978: 46)

Sin embargo, en términos formales sí es posible reconocer una solución unificadora entre todas las obras del centro cívico, inducida por una reducida gama volumétrica y material, en la que predominan los cuerpos blancos, las cubiertas con fuerte pendiente, las texturas rugosas y las aberturas resueltas a partir de perforaciones en los planos y plegado de muros para obviar el convencional recorte de ventanas. Las decisiones en torno a la materialidad se expresan con claridad. No se trataba de forzar 


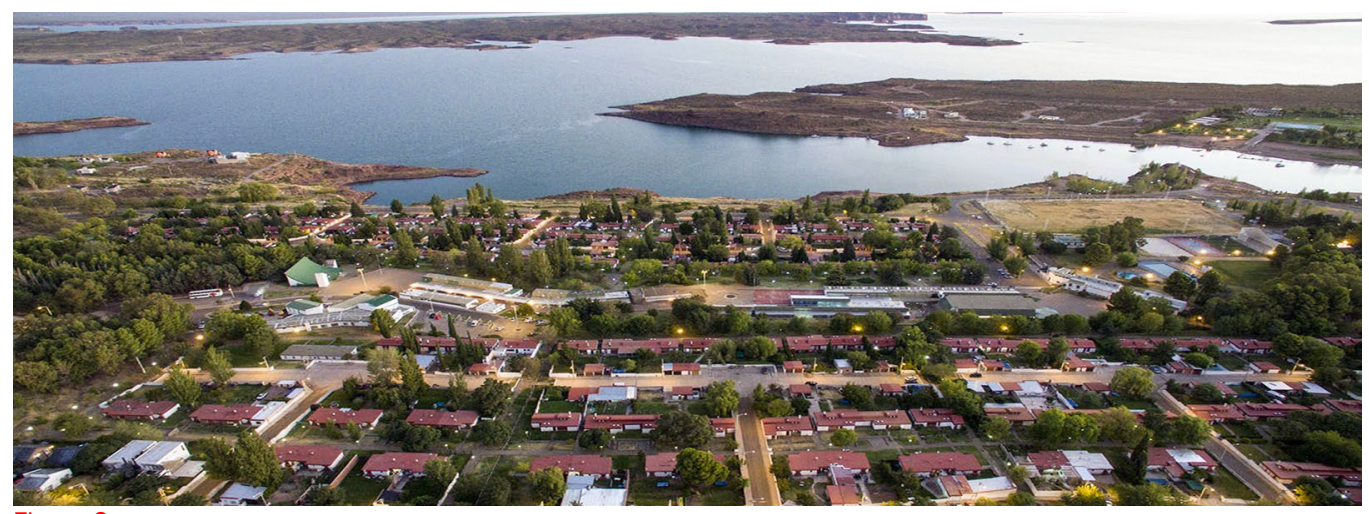

Figura 9.

Foto aérea de la Villa el Chocón (2016).

una "estética de la máquina", como había caracterizado Reyner Banham (1955) a la imagen de las obras de los arquitectos más difundidos de las primeras décadas del siglo $X X$, sino resaltar la naturaleza de los materiales elegidos mediante estrategias proyectuales específicas, reconsiderando el problema tecnológico en el contexto de la realidad nacional.

“La premisa fue el cuidado obsesivo en el uso y expresión de materiales, estructuras y elementos constructivos, dentro de una búsqueda de 'verdad ontológica' (intrínseca) en cada constituyente del vocabulario arquitectónico"

(Llauró y Urgell, 1973, p. 29).

En esta línea, el hormigón armado constituyó uno de los materiales más utilizados, tanto por costo, rapidez, ductilidad y estética.
La propuesta arquitectónica estaba acompañada por un atento diseño del mobiliario y de los objetos de uso cotidiano, tanto para los edificios públicos como para las viviendas. La empresa seleccionada en el concurso de antecedentes para realizar estas tareas, Stilka S.A.C.I., estuvo atenta a la experimentación con nuevos materiales, la tipificación de componentes y capacidad de traslado y apilamiento. "Se quiso respetar, a través del diseño y de las técnicas y materiales utilizados, el carácter de la arquitectura en la que se ambientan los elementos, evitando que entraran en colisión con los ámbitos, y buscando un clima cálido y afectivo que contrarrestara la aridez del paisaje" (Villa, 1970: 64). En relación al entorno físico, la intensa irrigación y la planificada forestación del área urbanizada formaron parte fundamental de la propuesta de apropiación social, buscando atenuar las condiciones de viento extremo y aridez que caracterizan a la región. 


\section{REFLEXIONES: NUEVAS IDEAS SOBRE LA CIUDAD Y LA ARQUITECTURA}

En la década de 1960 el proyecto para la Villa permanente el Chocón en la Provincia de Neuquén constituyó un referente insoslayable en materia urbanística y arquitectónica, no sólo por su escala -totalizando más de 40.000 m2 de obra construida- sino también por la relevancia y difusión que alcanzó en el debate disciplinar, en particular, y para la sociedad, en general.

“Por primera vez se nos planteó la necesidad de proyectar de una sola vez el hábitat físico de una pequeña comunidad. No sólo eso, sino trabajar en un área que fue el foco de atención de todo el país en su momento"

(Llauró y Urgell, 1978, p. 46)

En el marco de la mirada existencialista que por estos años había logrado un alto reconocimiento, la experiencia de los residentes constituyó una cuestión relevante para la disciplina, preocupando no sólo a proyectistas, sino que también formó parte de los intereses de una de las publicaciones que dio a conocer la obra. En 1973 la revista Nuestra Arquitectura, al cubrir la inauguración de la villa, realizó entrevistas a los habitantes, concluyendo que la propuesta arquitectónica viabiliza la generación de vida comunitaria, preservando un confortable nivel de privacidad.
La villa responde fielmente a las expectativas creadas... Los espacios públicos, los recorridos peatonales, las pérgolas, todo aquello tan caro a Ios arquitectos ¿son meros recursos o el usuario los percibe, los usa, los siente como parte de la cobertura que el entorno urbano debe brindar? $\mathrm{Si}$ nos atenemos a las respuestas veremos que sí, que los habitantes gozan los recorridos peatonales con absoluta soltura (Villa, 1973: 34).

El abordaje pormenorizado de la propuesta urbano-arquitectónica de Villa el Chocón que se propuso el presente artículo ha permitido identificar que el proyecto es elocuente de un "momento" en que la búsqueda de una alternativa a la homogeneidad urbana impuesta por el Movimiento Moderno así como la sensibilidad por los materiales en tanto expresión arquitectónica se constituyeron en el común denominador de una parte importante de las experiencias disciplinares renovadoras de las décadas de 1950 y 1960 (Solá Morales, 1991). Entre ellas también es posible mencionar el Cementerio Parque en Mar del Plata, Provincia de Buenos Aires (Horacio Baliero y Carmen Córdoba, 1961/1968), la Sede de la Asociación Rosarina de Intercambio Cultural Argentino Norteamericano en Rosario, Provincia de Santa Fe (Rufino de la Torre, Hilarión Hernández Larguía y Anibal Moliné, 1961/1963) y el Instituto de Previsión Social en Posadas, Provincia de Misiones (Mario Soto y Raúl Rivarola, 1959/1964). Como afirmaba Francisco Bullrich en relación a la producción 


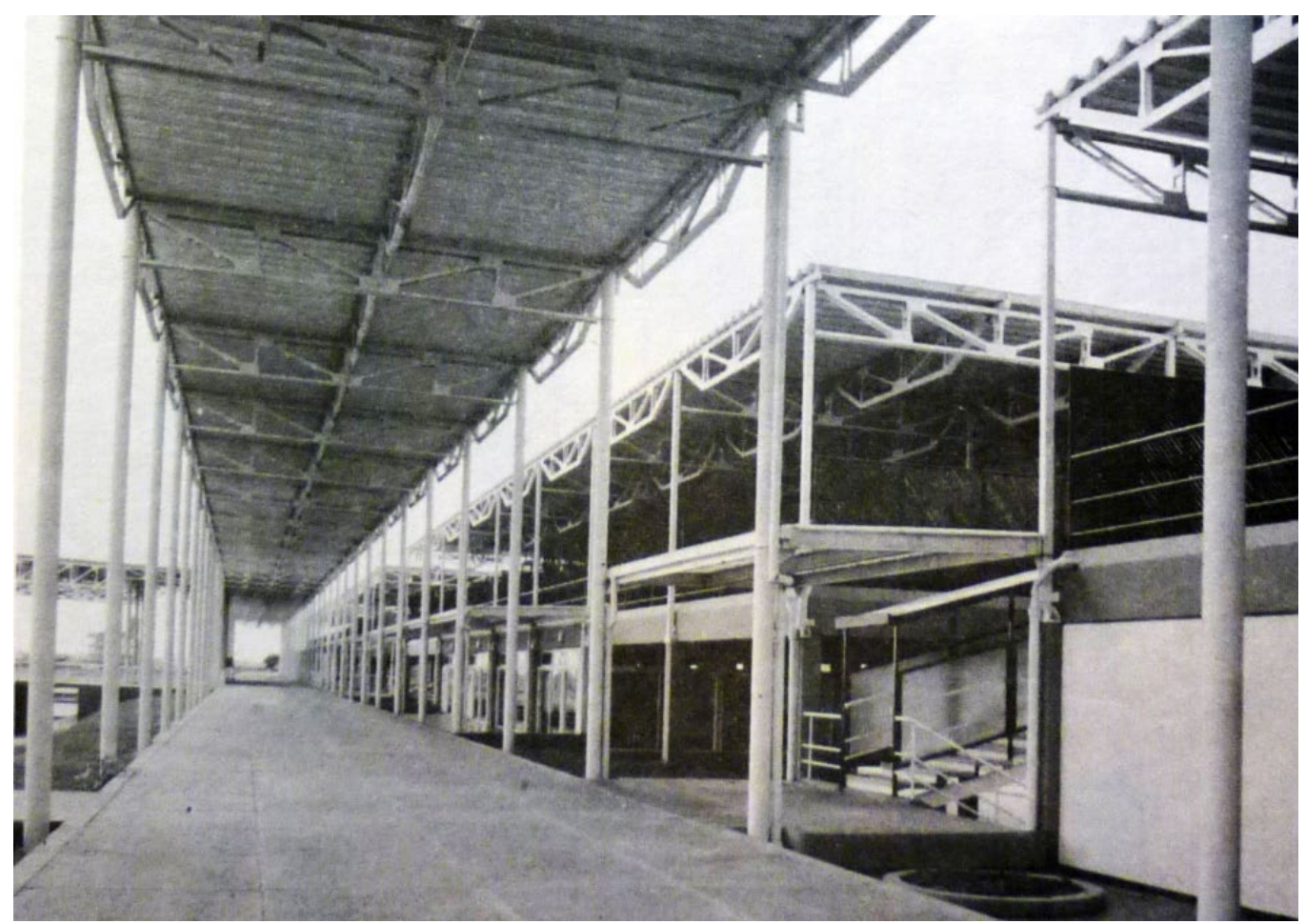

Figura 10

Calle peatonal del centro Cívico de la Villa Yacyretá (1980).

arquitectónica de estos años, estas obras “eran suficientemente buenas para ser publicadas en las revistas internacionales, y que la mayor parte de la producción argentina de ese momento estaba vinculada al trabajo y al estilo que se llevaba a cabo en el mundo" (1969: 37). Sin embargo, un nuevo posicionamiento disciplinar se definió en la década de 1970. Obras como la ciudad Nueva Federación en la Provincia de Entre Ríos (Carlos Viarenghi y Estela Iglesias, 1975/1979) o la Villa Permanente Yacyretá en la Provincia de Corrientes (Juan Manuel Llauró y Asociados,
Jorge Moscato y Rolando Schere 1975/1978) dejan de manifiesto un giro significativo en materia proyectual. La producción en serie, la racionalidad constructiva y la necesidad de controlar la totalidad del diseño confluyeron en desarrollar propuestas basada en la sistematización de módulos la previsión del crecimiento y los cambios de uso a futuro, y la exteriorización formal de los adelantos tecnológicos. De esta manera, se inicia así otro "momento", una alternativa de proyecto que ha sido identificada por la historiografía como "Arquitectura de Sistemas" (Aliata, 2014), y 
que encuentra sus fundamentos en debates alejados ya de la existencia humana y la fenomenología del lugar.

\section{BIBLIOGRAFÍA}

ALIATA, F. (2014, julio). Arqueología de la arquitectura de sistemas. Registros, 10(11) 6-19.

BANDIERI, S.; BLANCO, G. (2012). Promesas incumplidas: hidroenergía y desarrollo agrario regional en la norpatagonia argentina. Estudios Rurales. Publicación de Centro de Estudios de La Argentina Rural. 1(2), 148171. Disponible en línea http://ppct.caicyt. gov.ar/index.php/estudios-rurales/article/ view/1443/1341 Consultado el 26/03/2019.

BANHAM, R. (1955, abril). The machine aesthetic. Architectural Review, 118, 225-228.

BULLRICH, F (1969, octubre). Arquitectura argentina 1960/1970. Summa, 19, 37-82.

GIEDION. S. (1944). The need for a New Monumentality. En: Zucker, P. New architecture and city planning. New York, Philosophical Library, 549/568.

GIEDION. S. (1951). Reaffirmation of the aims of CIAM, Bridgewater 1947. A decade of new architecture. Zurich, Editions Girsberger, 16/17.

GUTIÉRREZ, R. (2004). Casas blancas: un momento de reflexión sobre la arquitectura argentina". Summa+, 63, 80/87.
IGLESIA, R. (1978). La reacción antirracionalista de las casas blancas. Buckminster Fuller, Archigram \& Co, La reacción antirracionalista de las casas blancas. Buenos Aires: Espacio Editora.

Introducción (1970, setiembre). Summa, 29, 28/30.

LIERNUR, J.F. (2001). Arquitectura en la Argentina del siglo XX. La construcción de la modernidad. Buenos Aires: Fondo Nacional de las Artes.

LLAURÓ, J. M.; URGELL, J. (1973). Notas sobre una experiencia en arquitectura religiosa. Nuestra Arquitectura, 482, 28-42.

LLAURÓ, J.M.; URGELL, J. (1978, octubre/ noviembre). Estudio Llauró-Urgell y Asociados. Summa, 129/130, 44-150.

MONTANER, J. M. (1997). La modernidad superada. Arquitectura, arte y pensamiento del siglo XX. Barcelona: Gustavo Gili.

MUMFORD, E. (2000). The CIAM discourse on urbanism, 1928 - 1960. Cambridge: MIT Press.

MUMFORD, L. (1937). The death of the monument. Circle: International. Survey of Constructive Art. London: Faber and Faber, $263 / 270$.

NUESTRA SEÑORA DE FÁTIMA (2005). En: González Montaner, B. (ed.). Vanguardias Argentinas. Obras y movimientos en el siglo XX. Arquitectura Contemporánea I, v. 3, Buenos Aires: Clarín, 34-41. 
NORBERG SCHULZ, C. (1979). Genius loci. Paesaggio, ambiente, architettura. Milán: Electra ed.

PLOTKIN, S. (2018, enero-junio). Grandes luces. Vivienda y arquitectura en el ciclo de producción de la energía eléctrica. Registros, 14(1), 126-140. Disponible en línea https:// revistasfaud.mdp.edu.ar/registros/article/ view/211/169 Consultado el 26/03/2019.

SCHERE, R (2008). Concursos 1826-2006. Buenos Aires: SCA.

SERT, J. L. (1955a). Prólogo a la edición en español. En: Tyrwhiff, J.; Sert, J. L.; Rogers, E. El corazón de la ciudad. Barcelona: Editorial Científico-médica, V-VI.

SERT, J. L. (1955b). Centros para la vida de la comunidad. En: Tyrwhiff, J.; Sert, J. L.; Rogers, E. El corazón de la ciudad. Barcelona: Editorial Científico-médica, 3-16.

SOLÁ- MORALES, I. (1991). Diferencias. Topografía de la arquitectura contemporánea. Barcelona: Gustavo Gili.

VAN DEN HEUVEL, D. (2017). The Lijnbaan in Rotterdam: A sound urban form against city disruption. En: Gosseye, J.; Avermaete, T. (eds.). Shopping Towns in Europe. Commercial Collectivity and the Architecture of the Shopping Centre, 1945-1975. London: Bloomsbury, 65-77.

VILLA EL CHOCÓN (1970, setiembre). Summa, (29), 50/67.

Villa Permanente de Yacyretá en Ituzaingó, Corrientes (1984, octubre). Summa, (205), $63 / 80$.
WILLIAMS, F. (2014a). Capitales extraviadas. Ciudad, equipamiento administrativo y monumentalidad en las nuevas provincias del Sur argentino. Estudios del Hábitat, 12(2), 115-130. Disponible en línea https://revistas. unlp.edu.ar/Habitat/article/view/1137/pdf Consultado el 26/03/2019.

WILLIAMS, F. (2014b). Urbanización y paisaje en la Patagonia argentina: interrogantes sobre los grandes emprendimientos energéticos y sus "villas permanentes". Registros, 11, 5771. Disponible en línea https://revistasfaud. mdp.edu.ar/registros/article/view/53/63 Consultado el 26/03/2019.

ZUCCARO MARCHI, L. (2013). The Heart of the City: Continuity and Complexity of an urban design concept. Tesis doctoral Istituto Universitario di Architettura di Venezia y Technische Universiteit Delf, inédita. Disponible en línea https://repository.tudelft. nl/islandora/object/uuid\%3A6f06d141e702-462f-a507-524762c7affO Consultado el 26/03/2019.

ZUCKER, P. (1944). Planning in three dimensions. New architecture and city planning. New York: Philosophical Library, 3/12. 


\section{FUENTES DE LAS IMÁGENES}

Figura 01: Plan Director para Buenos Aires (Le Corbusier, Jorge Ferrari Hardoy y Juan Kurchan, 1937/1938). Fuente: Liernur, 2001: 183

Figura 02: Vista peatonal del Distrito Lijnbaan en Rotterdam, 1965 (Jaap Bakema y Jo van der Broek, 1949/1953). Fuente: Van den Heuvel, 2017: 73 .

Figura 03: Interior de la Iglesia Nuestra Señora de Fátima en Martínez, Provincia de Buenos Aires, 2005 (Claudio Caveri y Eduardo Ellis, 1956/1959). Fuente: Nuestra Señora, 2005: 41.

Figura 04: Esquema general de la Villa el Chocón, 1968. Fuente: Llauró y Urgel, 1978: 67.

Figura 05: Sector residencial de la Villa el Chocón, vista desde el extremo Este del centro cívico, ca. 2008. Fuente: http://urgell-penedourgell.com/proyectos/categoria/003/villapermanente-el-chocon/

Figura 06: Planta del centro cívico de la villa, 1968. Fuente: Llauró y Urgel, 1978: 68.

Figura 07: Vista del centro cívico, circulación peatonal, 2007. Fuente: Williams, 2014a: 128.

Figura 08: Vista del atrio de la iglesia, 2010. Fuente: http://www1.rionegro.com.ar/ blog/eh/?mode=viewcat\&cat_id=1\&pageno $=125 \&$ limit $=5$
Figura 09: Foto aérea de la Villa el Chocón (2016). Fuente: https://www.neuqueninforma.gob. ar/villa-el-chocon-cumple-43-anos-de-vida-institucional/

Figura 10: Calle peatonal del centro Cívico de la Villa Yacyretá (1980). Fuente: Villa, 1984: 73. 
